\title{
Functional connection identified between Crohn's disease, activation of caspase and autophagy
}

A study in Nature has revealed the functional consequence of the Thr300Ala (T300A) variant of ATG16L1, an adaptor protein central to formation of autophagosomes, and confirms there is a connection between caspase activation, autophagy and Crohn's disease.

The A>G SNP (rs2241880) in ATG16L1 that encodes the T300A variant is strongly associated with Crohn's disease, but until now there was no conclusive evidence it was the causal allele on the risk haplotype.

Menno van Lookeren Campagne and colleagues performed a multiple sequence alignment analysis, identifying putative

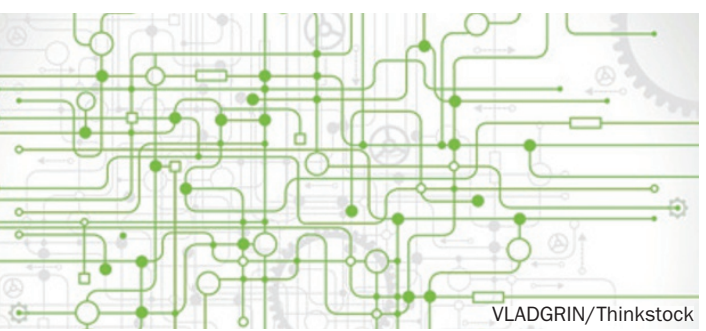

caspase target sequences in ATG16L1. Cleavage of the T300A variant was shown to be enhanced when in-vitro-translated ATG16L1 $\beta$ (one of the two major isoforms expressed in intestinal epithelium and macrophages) was incubated with recombinant active caspase 3; mutagenesis of the consensus caspase cleavage site at amino acids 296-299 prevented cleavage.

Cellular stress was found to have a role in caspase-mediated accelerated degradation of the T300A variant in cell lines and primary cells from human donors and ATG16L1 T316A knock-in mice (T316A being the mouse equivalent of T300A). Caspase 3 was confirmed as the ATG16L1 effector caspase.

The researchers also established that the T300A variant confers a defect in metabolic-stress-induced autophagy. In addition, clearance of the ileal pathogen Yersinia enterocolitica was shown to be defective in macrophages of T316A knock-in mice and that the defective clearance resulted in an inflammatory cytokine response.

So, enhanced ATG16L1 cleavage in the presence of T300A and persistent cellular stress results in diminished autophagy and the production of inflammatory cytokines, which the authors link to the generation of a chronic inflammatory state in the intestine.

"The identification of the sensitivity of this protein to caspase-3-mediated destruction may provide insight into how specific environmental exposures convert genetic disease risk into clinical symptoms," confirm Arthur Kaser and Richard Blumberg in a News \& Views article on the study.

Natalie J. Wood

Original article Murthy, A. et al. A Crohn's disease variant in Atg16/1 enhances its degradation by caspase 3. Nature 506, 456-462 (2014)

Further reading Kaser, A. \& Blumberg, R. S. Stressful genetics in Crohn's disease. Nature 506, 441-442 (2014) 